\title{
Avaliação de Ferramentas BPM: Uma Análise Comparativa de Soluções Comerciais
}

\author{
Marco Sousa ${ }^{1}$, Nuno Lopes ${ }^{1}$, Óscar Ribeiro ${ }^{1}$, Joaquim P. Silva ${ }^{1}$ \\ marcocruzsousa@gmail.com, nlopes@ipca.pt, oribeiro@ipca.pt, jpsilva@ipca.pt \\ ${ }^{1}$ Instituto Politécnico do Cávado e Ave, Vila Frescaínha S. Martinho, 4750-810, Barcelos, Portugal
}

DOI: $10.17013 /$ risti.35.70-85

\begin{abstract}
Resumo: A diversidade de ferramentas BPM torna cada vez mais difícil para uma organização realizar uma seleção adequada de uma ferramenta. Este artigo propõe uma estrutura para orientar a avaliação de ferramentas BPM, a qual tem como foco principal o ciclo de vida BPM e inclui um conjunto de critérios tecnológicos. Esta estrutura de avaliação foi utilizada para classificar uma edição comercial de quatro ferramentas BPM, segundo a sua documentação técnica. O resultado deste artigo apresenta uma análise comparativa com base no método AHP, a qual evidencia as principais diferenças entre as ferramentas BPM ao longo do ciclo de vida BPM. Pretende-se incentivar a adoção da gestão dos processos de negócio junto das organizações e ajudar as mesmas na escolha da ferramenta de BPM a utilizar.
\end{abstract}

Palavras-chave: Gestão de Processos de Negócio, Ciclo de Vida BPM, Ferramentas BPM.

\section{Evaluation of BPM Tools: A Comparative Analysis of Commercial Solutions}

\begin{abstract}
The diversity of BPM tools makes it increasingly difficult for an organization to make a proper selection of a tool. This article proposes a structure to guide the evaluation of BPM tools, which has as main target the BPM lifecycle and includes a set of technological criteria. This evaluation framework was used to classify a commercial edition of four BPM tools according to their technical documentation. The result of this article presents a comparative analysis based on the AHP method, which highlights the main differences between BPM tools throughout the BPM lifecycle. The aim is to encourage the adoption of business process management by organizations and help them in the choice of the BPM tool to use.
\end{abstract}

Keywords: Business Process Management, BPM Lifecycle, BPM Tools.

\section{Introdução}

As organizações devem ser eficientes, os processos devem ser constantemente avaliados e melhorados, como parte das operações diárias do negócio. Um processo de negócio pode 
ser definido como um conjunto de atividades que são executadas em coordenação num ambiente organizacional e técnico. Essas atividades realizam em conjunto o objetivo do negócio (Weske, 2012). Os processos de negócio foram muitas vezes categorizados como processos de gestão, operacionais ou de suporte, sendo os processos operacionais aqueles que estão relacionados com a atividade central do negócio em termos de produção ou fornecimento de serviços com valor acrescentado aos clientes (Hook, 2011). A gestão de processo de negócio (BPM) é uma disciplina de gestão que requer uma mudança de pensamento centrada no processo (Hill, Sinur, Flint, \& Melenovsky, 2006). BPM suporta os processos de negócio através da utilização de métodos, técnicas e sistemas de software, para desenhar, representar, controlar e analisar processos operacionais, envolvendo pessoas, organizações, aplicações, documentos e outras fontes de informação (Van der Aalst, ter Hofstede, \& Weske, 2003). O ciclo de vida BPM é de grande importância para levar a cabo uma melhoria contínua dos processos, contribuindo para que as organizações procurem ferramentas que proporcionem o apoio necessário na realização dessas melhorias (Peralta, Salgado, Montejano, \& Riesco, 2016). Devido à diversidade de ferramentas BPM, selecionar qual a ferramenta mais adequada para uma organização revela-se uma tarefa cada vez mais difícil. Com a adoção de BPM as organizações podem obter benefícios, tais como, uma maior visibilidade e conhecimento das atividades, uma maior habilidade em identificar problemas e uma melhor definição de regras e funções do negócio (Ko, 2009). BPM tem um papel ambicioso na melhoria contínua dos processos e em promover a sua eficácia e eficiência (Rademakers, 2012). BPM contribui para uma gestão mais eficiente dos processos e, com ferramentas específicas, as organizações poderão alcançar os seus objetivos organizacionais.

Este artigo propõe uma estrutura para orientar a avaliação de ferramentas BPM, a qual tem como foco principal o ciclo de vida BPM e inclui um conjunto de critérios tecnológicos. Esta estrutura de avaliação foi utilizada para classificar uma edição comercial de quatro ferramentas BPM, segundo a sua documentação técnica. O resultado deste artigo apresentará uma análise comparativa, com base no método AHP, entre as quatro ferramentas BPM.

Este artigo está estruturado em cinco secções. Na secção 2 contextualiza-se o termo "ciclo de vida BPM" e são introduzidos outros estudos relacionados com a avaliação de ferramentas BPM. Na secção 3 descreve-se a estrutura de avaliação constituída por um conjunto de critérios tecnológicos, identifica-se e classifica-se as ferramentas BPM. Na secção 4 avalia-se as ferramentas BPM de acordo com os resultados obtidos. Na secção 5 retira-se uma conclusão na qual se pretende sintetizar todo o trabalho realizado.

\section{Estado da Arte}

O exato significado de BPM está diretamente relacionado com o seu ciclo de vida (Weske, 2012). O ciclo de vida BPM demonstra que a implementação de processos de negócio é um plano contínuo de melhoria devido à frequente mudança do ambiente empresarial e à necessidade de otimização dos processos (Rademakers, 2012). Na literatura científica existem várias propostas com diferentes visualizações, definições e perspetivas de um ciclo de vida BPM: (i) Van der Aalst, ter Hofstede \& Weske (2003) defendem um ciclo de vida BPM restrito aos processos de negócio operacionais, (ii) Hill, Sinur, Flint \& 
Melenovsky (2006) defendem um ciclo de vida BPM que contribuiu para criar valor para um processo de negócio, (iii) Rademakers (2012) defende um ciclo de vida BPM com cinco fases essenciais para criar um processo de negócio totalmente funcional, (iv) Weske (2012) defende um ciclo de vida BPM que envolve a Administração e os Intervenientes do processo no centro de todo o ciclo de vida BPM e (v) Van der Aalst (2013) defende um ciclo de vida BPM que tem como foco principal dois tipos de análise distintos, um tipo baseado no modelo e outro tipo baseado nos dados do processo de negócio. A proposta do ciclo de vida BPM referenciada por Weske (2012) terá um papel fundamental no desenvolvimento da estrutura de avaliação, uma vez que constitui a proposta que melhor se enquadra neste artigo. Nesta proposta, o ciclo de vida BPM (i) é direcionado aos processos organizacionais (processos que caracterizam as funcionalidades do negócio), (ii) inclui subfases e (iii) tem como foco central os intervenientes do processo de negócio.

Nos últimos anos têm sido publicados diferentes estudos relacionados com a avaliação de ferramentas BPM: (i) Hill \& Sinur (2010) apresentam um relatório técnico designado, por "Magic Quadrant for Business Process Management Suites", elaborado pela consultora Gartner, que inclui uma avaliação das ferramentas BPM comerciais de vinte e cinco distribuidores; (ii) Craggs (2011) apresenta um relatório técnico, elaborado pela consultora Lustratus, que inclui uma avaliação das ferramentas BPM comerciais de três distribuidores; (iii) Richardson \& Miers (2013) apresentam um relatório técnico, designado por “The Forrester Wave: BPM Suites, Q1 2013", elaborado pela consultora Forrester, que inclui uma avaliação das ferramentas BPM comerciais de dez distribuidores; (iv) Bischoff \& van Dinther (2016) apresentam uma análise comparativa de quatro ferramentas BPM open source, onde a avaliação das ferramentas é realizada com base num conjunto de critérios e na implementação de diferentes processos de negócio; (v) Meidan, García-García, Escalona \& Ramos (2016) apresentam uma avaliação de sete ferramentas BPM open source, utilizando uma metodologia que combina a revisão sistemática da literatura (SLR) com modelos de qualidade, e tendo por base um conjunto de critérios tecnológicos subdivididos pelas fases do ciclo de vida BPM e um esquema de classificação; e (vi) Dunie, Kerremans, Baker \& Wong (2017) apresentam um relatório técnico, designado por "Magic Quadrant for Intelligent Business Process Management Suites", elaborado pela consultora Gartner, que inclui uma avaliação das ferramentas BPM comerciais de dezoito distribuidores. Todos estes estudos são um ponto de partida para personalizar outras estruturas de avaliação, permitindo adicionar critérios tecnológicos, utilizar diferentes ciclos de vida BPM, definir novos métodos de classificação e avaliar diferentes edições das ferramentas BPM.

\section{Método de Avaliação}

A estrutura definida para orientar a avaliação das ferramentas BPM tem como foco principal o ciclo de vida BPM referenciado por Weske (2012), com o intuito de explorar as necessidades mais específicas das organizações, e inclui um conjunto de critérios tecnológicos referenciados na literatura científica que estarão na base da método de avaliação. O ciclo de vida BPM referenciado por Weske (2012) é constituído pelas seguintes fases: (i) Desenho e Análise, (ii) Configuração, (iii) Execução e (iv) Avaliação, incluindo $(v)$ Administração e Intervenientes como foco central de todo o ciclo de vida BPM. Neste artigo também foram atribuídos critérios tecnológicos em Administração e 
Intervenientes apesar de esta não ser uma fase explicita do ciclo de vida BPM, mas sim um conjunto de tarefas de suporte às fases do ciclo de vida BPM.

\subsection{Fase de Desenho e Análise}

Na fase de Desenho e Análise, inicia-se o ciclo de vida BPM, na qual são realizadas pesquisas sobre processos de negócio e o seu ambiente técnico e organizacional. Esta fase é constituída pelas seguintes subfases: (i) subfase de Identificação e Modelação Processo Negócio, (ii) subfase de Validação, (iii) subfase de Simulação e (iv) subfase de Verificação.

Na subfase de Identificação e Modelação Processo Negócio, e com base nas pesquisas, os processos de negócio são identificados, revistos, validados e representados em modelos de processos de negócio (Weske, 2012). Após identificar o processo de negócio, pode ser utilizado um vasto conjunto de linguagens de modelação para expressar os modelos de processo de negócio, tais como BPMN ou UML (OMG, 2011). Neste contexto, a interoperabilidade entre as diferentes linguagens de modelação é um aspeto muito importante nos sistemas BPM. O modelo formal do processo de negócio deve ser representado em diferentes perspetivas para que os diferentes intervenientes o possam analisar e entender sem ambiguidades. A disponibilidade para gerar documentação sobre os processos de negócio é também um aspeto importante para a comunicação entre os intervenientes (Meidan et al., 2016). Outro aspeto importante dentro da modelação de processos de negócio é a utilização de regras de negócio que permitem incluir na modelação do processo tipos de pontos de decisão flexíveis do negócio (Rademakers, 2012). Muitas ferramentas de modelação de processos de negócio fornecem repositórios de modelos de processos que permitem o armazenamento e recuperação de modelos de processos (Van der Aalst, 2013). Em BPMN 2.0 a reutilização pode estar presente nos subprocessos, pois estes podem ser reutilizados por outros processos de negócio (Rademakers, 2012). A fim de detetar os aspetos a melhorar no processo e, desta forma, promover a sua melhoria contínua, são definidas métricas que são chamadas de indicadores de desempenho do processo (KPI) (Peralta et al., 2016). Na subfase de Validação, um instrumento útil utilizado na validação de um processo de negócio é o workshop, durante o qual as pessoas envolvidas discutem o processo. Os participantes do workshop vão verificar se todas as instâncias válidas do processo de negócio estão refletidas no modelo de processo de negócio. Na subfase de Simulação, os processos de negócio são simulados, o que permite aos intervenientes navegarem pelo processo passo a passo e verificarem se o processo atualmente expõe o comportamento desejado (Weske, 2012). Na subfase de Verificação, os processos de negócio são analisados para verificar se funcionam corretamente. Quando existem processos a serem melhorados, a análise a esses processos é realizada antes que as alterações sejam colocadas em vigor (Van der Aalst \& van Hee, 2004). A Tabela 1 resume o conjunto de critérios da fase de Desenho e Análise.

\begin{tabular}{ll}
\hline Critérios & Descrição \\
\hline \#DA.1 & Suporte para diferentes linguagens modelação de processos. \\
\hline \#DA.2 & Suporte para interoperabilidade entre linguagens de modelação de processos. \\
\hline \#DA.3 & Suporte para diferentes perspetivas dos processos. \\
\hline \#DA.4 & Suporte para gerar documentação dos processos. \\
\hline
\end{tabular}




\begin{tabular}{ll}
\hline Critérios & Descrição \\
\hline \#DA.5 & Suporte para modelação de processos utilizando regras de negócio. \\
\hline \#DA.6 & Suporte para reutilização de modelos de processos. \\
\hline \#DA.7 & Suporte para modelação de processos utilizando KPI. \\
\hline \#DA.8 & Suporte para sugestões de melhoria dos processos. \\
\hline \#DA.9 & Suporte para simulação dos processos. \\
\hline \#DA.10 & Suporte para verificação dos processos. \\
\hline
\end{tabular}

Tabela 1 - Critérios Tecnológicos Fase de Desenho e Análise

\subsection{Fase de Configuração}

$\mathrm{Na}$ fase de Configuração, após o modelo do processo de negócio ser desenhado e verificado, é necessário implementar o processo de negócio (Weske, 2012). Um modelo de processo de negócio é composto por papéis dos utilizadores, definição de estados e transições entre esses mesmos estados. Os modelos de processos de negócio têm o propósito de representar uma realidade organizacional e são implementados pelas instâncias de processos de negócio (Marques \& Guerreiro, 2016). Esta fase é constituída pelas seguintes subfases: (i) subfase de Seleção Sistema, (ii) subfase de Implementação e (iii) subfase de Testes e Implantação.

Na subfase de Seleção Sistema, a tradução de um modelo gráfico num formato executável poderá ser efetuada de forma automática e o modelo do processo será melhorado com informação técnica o que facilitará a execução do processo no sistema BPM. O modelo executável de um processo de negócio é modelado por linguagens executáveis de processos de negócio para Web Services, tais como WS-BPEL. A linguagem de modelação BPMN fornece um mapeamento para a linguagem de execução WS-BPEL, mas nem todas as linguagens de modelação mapeiam as linguagens de execução (OMG, 2011). Após a tradução do modelo do processo em um modelo executável do processo de negócio será possível adicionar um acordo de nível de serviço (SLA) ao modelo associado ao KPI, o que facilitará a monitorização do processo de negócio (Meidan et al., 2016). Na subfase de Implementação, a interação dos utilizadores com o sistema pode ser efetuada através de interfaces gráficas (Weske, 2012). A gestão de utilizadores deve ser implementada no sistema BPM, permitindo definir quais os papéis dos diversos utilizadores com base as suas capacidades. Os papéis de cada utilizador normalmente estão descritos na estrutura organizacional das organizações e, normalmente são armazenados em sistemas do tipo LDAP. A configuração de um sistema BPM envolve aspetos transacionais, a implementação de mecanismos para o tratamento de exceções e o controlo das transações (Meidan et al., 2016). Na subfase de Testes e Implantação, a implementação do processo de negócio deve ser testada, os testes de integração e desempenho são importantes para detetar potenciais problemas de execução. Após a realização dos testes, o sistema deve ser implantado no ambiente para o qual foi desenvolvido. Dependendo de opções específicas, podem ser requeridas atividades adicionais como, por exemplo, o treino de pessoas ou a migração dos dados de uma aplicação para a nova plataforma (Weske, 2012). Concluída a implantação do sistema, os processos estão disponíveis para todos os utilizadores finais, podendo ser 
conectados e integrados com outros serviços e recursos, internos ou externos, tais como REST ou WSDL (Meidan et al., 2016). A Tabela 2 resume o conjunto de critérios da fase de Configuração.

\begin{tabular}{ll}
\hline Critérios & Descrição \\
\hline \#CF.1 & Suporte para linguagens de execução de processos. \\
\hline \#CF.2 & Suporte para tradução manual ou automática num modelo executável do processo. \\
\hline \#CF.2 & Suporte para adição de SLA e conexão com um KPI. \\
\hline \#CF.4 & Suporte para desenho de interfaces gráficas. \\
\hline \#CF.5 & Suporte para diferentes linguagens de programação para implementar serviços. \\
\hline \#CF.6 & Suporte para atribuir papéis aos utilizadores. \\
\hline \#CF.7 & Suporte para descrição dos papéis dos utilizadores. \\
\hline \#CF.8 & Suporte para a importação de uma estrutura organizacional de outro sistema. \\
\hline \#CF.9 & Suporte para o tratamento de exceções e o controlo das transações nos processos. \\
\hline \#CF.10 & Suporte para execução distribuída. \\
\hline \#CF.11 & Suporte para integração com outros sistemas e serviços.
\end{tabular}

Tabela 2 - Critérios Tecnológicos da Fase de Configuração

\subsection{Fase de Execução}

Na fase de Execução, as instâncias do processo de negócio são inicializadas para preencher os objetivos do negócio das organizações. A execução do processo precisa atender a uma correta orquestração do processo, garantindo que as atividades dos processos são desempenhadas de acordo com as restrições de execução especificadas no modelo do processo (Weske, 2012). Os utilizadores têm um papel ativo e autónomo na execução dos processos de negócio, existindo por esta razão o risco de que as prescrições dos processos de negócio não sejam cumpridas em conformidade com as iniciais (Marques \& Guerreiro, 2016). Esta fase é constituída pelas seguintes subfases: (i) subfase de Operação, (ii) subfase de Monitorização e (iii) subfase de Manutenção.

Na subfase de Operação, os utilizadores interagem com as instâncias do processo de negócio através de interfaces gráficas para executar as tarefas, fornecer dados ao processo, obter e submeter documentos entre outras atividades. Neste sentido, a gestão documental ou um calendário podem ser implementados internamente no sistema BPM. Uma gestão de versões possibilita a execução em simultâneo de diferentes versões do mesmo processo e assim rastrear todas as versões em execução (Meidan et al., 2016). A subfase de Monitorização está relacionada com o controlo dos processos de negócio, na qual o objetivo é permitir que a operação das instâncias seja conduzida, usando um esforço limitado, para um estado estável previamente definido pela organização, sendo capaz de reagir às alterações que vão ocorrendo (Marques \& Guerreiro, 2016). Através desta monitorização é possível obter informação para o cálculo de KPI, a qual pode ser apresentada em painéis de visualização. Recorrendo a técnicas de visualização, um sistema BPM permite apresentar a informação de monitorização em diferentes vistas (Meidan et al., 2016). Na subfase de Manutenção, um sistema BPM disponibiliza a informação detalhada do estado atual das instâncias do processo de negócio (Weske, 
2012). Esta funcionalidade possibilita melhorar a previsão de problemas e riscos durante a execução dos processos. Outro aspeto importante num sistema BPM prendese com a capacidade de alterar os papéis das atividades ou recursos para equilibrar as cargas de trabalho (Meidan et al., 2016). A Tabela 3 resume o conjunto de critérios da fase de Execução.

\begin{tabular}{ll}
\hline Critérios & Descrição \\
\hline \#EX.1 & Suporte para gestão documental e de um calendário. \\
\hline \#EX.2 & Suporte para informar os utilizadores sobre as suas tarefas. \\
\hline \#EX.3 & Suporte para gestão de versões do modelo dos processos. \\
\hline \#EX.4 & Suporte para controlo e monitorização técnica. \\
\hline \#EX.5 & Suporte para diferentes vistas da informação de monitorização. \\
\hline \#EX.6 & Suporte para painéis de visualização e relatórios. \\
\hline \#EX.7 & Suporte para alterar o recurso de uma atividade numa instância do processo. \\
\hline \#EX.8 & Suporte para alterar regras de negócio dos processos. \\
\hline \#EX.9 & Suporte para detetar e resolver falhas. \\
\hline \#EX.10 & Suporte para alterar o equilíbrio da carga de trabalho entre os utilizadores. \\
\hline
\end{tabular}

Tabela 3 - Critérios Tecnológicos da Fase de Execução

\subsection{Fase de Avaliação}

Na fase de Avaliação, através da informação dos ficheiros de registo, é possível avaliar e melhorar os modelos de processo de negócio e as suas implementações. As técnicas de mineração de processos e monitorização da atividade do negócio (BAM) contribuem para identificar a qualidade do modelo do processo de negócio e a sua adequação ao ambiente de execução (Weske, 2012). As técnicas de mineração de processos permitem descrever um conjunto de análises que exploram a informação armazenada em registos de transações ou em sistemas de base de dados (Van der Aalst, 2013). A disponibilidade dos ficheiros de registo, outros recursos históricos como sistemas de base de dados e os níveis de negócio a atingir pelos valores definidos nos KPI são aspetos muito importantes num sistema BPM (Meidan et al., 2016). A Tabela 4 resume o conjunto de critérios para a fase de Avaliação.

\begin{tabular}{ll}
\hline Critérios & Descrição \\
\hline \#AV.1 & Suporte para técnicas de Mineração de Processos. \\
\hline \#AV.2 & Suporte para técnicas de Monitorização da Atividade do Negócio. \\
\hline \#AV.3 & Suporte para diferentes tipos de dados históricos. \\
\hline
\end{tabular}

Tabela 4 - Critérios Tecnológicos da Fase de Avaliação

\subsection{Administração e Intervenientes}

Em Administração e Intervenientes, nos diferentes níveis de abstração de um sistema BPM, existem inúmeros artefactos que devem ser bem organizados e estruturados 
(Weske, 2012). A diversidade de conteúdos no apoio à interação dos intervenientes é um aspeto muito relevante num sistema BPM (Meidan et al., 2016). A Tabela 5 resume o conjunto de critérios para Administração e Intervenientes.

\begin{tabular}{ll}
\hline Critérios & Descrição \\
\hline \#AI.1 & Suporte para diferentes tipos de documentação técnica da ferramenta BPM. \\
\hline \#AI.2 & Suporte para visualizar a maturidade da ferramenta BPM. \\
\hline \#AI.3 & Suporte para diferentes tipos de apoio técnico à ferramenta BPM. \\
\hline
\end{tabular}

Tabela 5 - Critérios Tecnológicos: Administração e Intervenientes

\subsection{Seleção das Ferramentas BPM}

Neste artigo optou-se por utilizar as seguintes ferramentas BPM: (i) Bonita ${ }^{1}$, (ii) ProcessMaker $^{2}$, (iii) Bizagi ${ }^{3}$ e (iv) Alfresco Process Services ${ }^{4}$.

A edição open source/freeware destas ferramentas BPM foi estudada por Sousa, Lopes, Ribeiro \& Silva (2018) na implementação de um processo de negócio ao longo da fase de Desenho e Análise. Estas ferramentas BPM surgem bem referenciadas em diversos estudos publicados na literatura científica relacionados com a avaliação de ferramentas BPM, que disponibilizam diversas edições open source/freeware e comerciais. Enquanto a edição open source/freeware suporta apenas uma das fases do ciclo de vida BPM, as edições comerciais suportam todo o ciclo de vida BPM. A Tabela 6 apresenta a designação da edição comercial, e respetiva versão, de cada uma das ferramentas BPM consideradas.

\begin{tabular}{ll}
\hline Ferramentas BPM & Edição Comercial - Versão \\
\hline Bonita & Performance - versão 7.5 \\
\hline ProcessMaker & Enterprise - versão 3.2 \\
\hline Bizagi & Modeler - versão $3.1 \mid$ Studio - versão 11.1 \\
\hline Alfresco Process Services & Enterprise - versão 1.7 \\
\hline
\end{tabular}

Tabela 6 - Edições Comerciais das Ferramentas BPM

\subsection{Método de Classificação}

O método de classificação desenvolvido neste artigo atribuirá em cada fase do ciclo de vida BPM uma classificação final, a qual permitirá uma avaliação mais concreta das funcionalidades de cada uma das ferramentas BPM. A Tabela 7 apresenta os níveis de pontuação definidos no método de classificação.

\footnotetext{
${ }^{1}$ Disponível em: https://www.bonitasoft.com/

${ }^{2}$ Disponível em: https://www.processmaker.com/

${ }^{3}$ Disponível em: https://www.bizagi.com/

${ }^{4}$ Disponível em: https://www.alfresco.com/bpm-software/
} 


\begin{tabular}{lll}
\hline No Pontos & Descrição & Símbolos \\
\hline o Pontos & Se a ferramenta BPM não suporta o critério de avaliação. & $\diamond \diamond$ \\
\hline 1 Ponto & Se a ferramenta BPM suporta o critério de avaliação (nível mínimo). & $\diamond$ \\
\hline 2 Pontos & $\begin{array}{l}\text { Se a ferramenta BPM suporta o critério de avaliação (nível mínimo) e } \\
\text { inclui outros aspetos relevantes para o critério de avaliação. }\end{array}$ & $\diamond \diamond$ \\
\hline
\end{tabular}

Tabela 7 - Níveis de Pontuação do Método de Classificação

\subsection{Classificação das Ferramentas BPM}

A classificação das ferramentas BPM teve por base a documentação técnica disponibilizada por cada uma das ferramentas BPM. Os resultados obtidos por cada uma das ferramentas BPM ao longo das fases do ciclo de vida BPM serão apresentados nas tabelas 8 a 12. A Tabela 8 resume os resultados obtidos pelas ferramentas BPM ao longo da fase de Desenho e Análise.

\begin{tabular}{|c|c|c|c|c|}
\hline Critérios & Bonita & ProcessMaker & Bizagi & Alfresco \\
\hline$\# D A .1$ & $\diamond \diamond$ & $\diamond \diamond$ & $\diamond$ & $\diamond \diamond$ \\
\hline$\# D A .2$ & $\diamond$ & $\diamond \diamond$ & $\diamond$ & $\diamond \diamond$ \\
\hline$\# D A .3$ & $\diamond$ & $\diamond$ & $\diamond$ & $\diamond$ \\
\hline$\# D A .4$ & $\diamond$ & $\diamond$ & $\diamond$ & $\diamond$ \\
\hline$\# D A .5$ & $\diamond \diamond$ & $\bullet$ & $\diamond$ & $\diamond \diamond$ \\
\hline \#DA.6 & $\bullet$ & $\diamond$ & $\diamond$ & $\diamond \diamond$ \\
\hline$\# D A .7$ & $\diamond$ & $\diamond$ & $\bullet$ & $\diamond \diamond$ \\
\hline$\# D A .8$ & $\diamond \diamond$ & $\diamond \diamond$ & $\bullet$ & $\diamond \diamond$ \\
\hline$\# D A .9$ & $\diamond$ & $\diamond \diamond$ & $\diamond$ & $\diamond \diamond$ \\
\hline$\# D A .1 O$ & $\diamond$ & $\diamond$ & $\diamond$ & $\diamond$ \\
\hline
\end{tabular}

Tabela 8 - Classificação da Fase de Desenho e Análise

Na fase de Desenho e Análise, ProcessMaker não disponibiliza informação técnica sobre o suporte para simulação de um processo de negócio. As quatro ferramentas BPM suportam diferentes perspetivas, permitem gerar diferentes tipos de documentação e incluem a funcionalidade de verificação de um processo de negócio. A Tabela 9 resume os resultados obtidos das ferramentas BPM na fase de Configuração.

\begin{tabular}{|c|c|c|c|c|}
\hline Critérios & Bonita & ProcessMaker & Bizagi & Alfresco \\
\hline$\# C F .1$ & $\diamond \diamond$ & $\diamond \diamond$ & $\diamond \diamond$ & $\diamond \diamond$ \\
\hline$\# C F .2$ & $\diamond$ & $\diamond$ & $\diamond$ & $\diamond$ \\
\hline$\# C F .3$ & $\diamond \diamond$ & $\diamond \diamond$ & $\diamond$ & $\diamond$ \\
\hline$\# C F .4$ & $\diamond$ & $\diamond$ & $\diamond \diamond$ & $\diamond \diamond$ \\
\hline$\# C F .5$ & $\diamond \diamond$ & $\bullet$ & $\diamond \diamond$ & $\leftrightarrow$ \\
\hline$\# C F .6$ & $\bullet$ & $\diamond \diamond$ & $\bullet$ & $\diamond$ \\
\hline$\# C F .7$ & $\diamond \diamond$ & $\diamond \diamond$ & $\diamond \diamond$ & $\diamond \diamond$ \\
\hline
\end{tabular}




\begin{tabular}{|c|c|c|c|c|}
\hline Critérios & Bonita & ProcessMaker & Bizagi & Alfresco \\
\hline \#CF.8 & $\diamond \diamond$ & $\diamond$ & $\diamond$ & $\diamond$ \\
\hline \#CF.9 & $\diamond$ & $\diamond \diamond$ & $\diamond$ & $\diamond$ \\
\hline$\# C F .10$ & $\diamond$ & $\diamond \diamond$ & $\diamond$ & $\diamond$ \\
\hline$\# C F .11$ & $\bullet$ & $\diamond$ & $\bullet$ & $\bullet$ \\
\hline
\end{tabular}

Tabela 9 - Classificação da Fase de Configuração

Na fase de Configuração, ProcessMaker é a única ferramenta que suporta linguagens de execução (funções ProcessMaker PHP). Bonita é a única ferramenta que não suporta a adição de SLA e uma conexão com um KPI. As quatro ferramentas BPM suportam a tradução manual ou automática num modelo executável de um processo de negócio e suportam a integração com outros sistemas e serviços (REST API, Web Services). A Tabela 10 resume os resultados obtidos das ferramentas BPM na fase de Execução.

\begin{tabular}{|c|c|c|c|c|}
\hline Critérios & Bonita & ProcessMaker & Bizagi & Alfresco \\
\hline$\# E X .1$ & $\bullet$ & $\diamond$ & $\diamond$ & $\diamond$ \\
\hline$\# E X .2$ & $\diamond \diamond$ & $\diamond$ & $\diamond$ & $\diamond \diamond$ \\
\hline$\# E X .3$ & $\leftrightarrow$ & $\diamond \diamond$ & $\diamond$ & $\leftrightarrow$ \\
\hline$\# E X .4$ & $\diamond$ & $\diamond \diamond$ & $\diamond$ & $\diamond$ \\
\hline$\# E X .5$ & $\diamond$ & $\diamond \diamond$ & $\diamond$ & $\diamond$ \\
\hline$\# E X .6$ & $\diamond \diamond$ & $\diamond$ & $\diamond$ & $\diamond$ \\
\hline$\# E X .7$ & $\diamond$ & $\diamond$ & $\diamond$ & $\diamond$ \\
\hline$\# E X .8$ & $\diamond \diamond$ & $\diamond \diamond$ & $\diamond$ & $\diamond \diamond$ \\
\hline$\# E X .9$ & $\diamond \diamond$ & $\diamond \diamond$ & $\diamond$ & $\diamond \diamond$ \\
\hline \#EX.10 & $\leftrightarrow$ & $\leftrightarrow$ & $\diamond$ & $\leftrightarrow$ \\
\hline
\end{tabular}

Tabela 10 - Classificação da Fase de Execução

Na fase de Execução, ProcessMaker é a única ferramenta que não disponibiliza a gestão de versões de um modelo de processo de negócio. Bonita e Alfresco não suportam a alteração de regras de negócio de um processo. As quatro ferramentas BPM disponibilizam gestão documental e de um calendário, suportam a alteração de um papel ou recurso em uma atividade de uma instância do processo de negócio e suportam a alteração do equilíbrio da carga de trabalho entre os utilizadores. A Tabela 11 resume os resultados obtidos das ferramentas BPM na fase de Avaliação.

\begin{tabular}{lcccc}
\hline Critérios & Bonita & ProcessMaker & Bizagi & Alfresco \\
\hline$\# A V .1$ & $\diamond \bullet$ & $\diamond \diamond$ & $\diamond \diamond$ & $\diamond \diamond$ \\
\hline$\# A V .2$ & $\diamond \bullet$ & $\diamond \diamond$ & $\diamond \diamond$ & $\diamond \diamond$ \\
\hline$\# A V .3$ & $\diamond \bullet$ & $\diamond \diamond$ & $\diamond \diamond$ & $\diamond \diamond$ \\
\hline
\end{tabular}

Tabela 11 - Classificação da Fase de Avaliação 
Na fase de Avaliação, Alfresco não disponibiliza informação técnica sobre o suporte para técnicas de Mineração de Processos e para técnicas BAM. As quatro ferramentas BPM suportam diferentes tipos de dados de histórico (ficheiros de registo, registo de eventos). A Tabela 12 resume os resultados obtidos das ferramentas BPM em Administração e Intervenientes.

\begin{tabular}{|c|c|c|c|c|}
\hline Critérios & Bonita & ProcessMaker & Bizagi & Alfresco \\
\hline$\# A I .1$ & $\diamond$ & $\diamond$ & $\diamond$ & $\diamond \diamond$ \\
\hline$\# A I .2$ & $\diamond$ & $\diamond$ & $\diamond$ & $\diamond$ \\
\hline \#AI.3 & $\diamond$ & $\diamond$ & $\diamond$ & $\diamond$ \\
\hline
\end{tabular}

Tabela 12 - Classificação de Administração e Intervenientes.

Em Administração e Intervenientes, as quatro ferramentas BPM disponibilizam o ano e número da edição de cada versão de lançamento, assim como diferentes tipos de suporte técnico à ferramenta (Fóruns, Suporte Técnico).

\section{Análise de Resultados}

A análise de resultados será de acordo com o conjunto de critérios tecnológicos definidos para o ciclo de vida BPM, a qual implica considerar múltiplos critérios tecnológicos. Por este motivo e com o objetivo de aprofundar mais esta análise de resultados, será utilizado o método Analytic Hierarchy Process (AHP) recorrendo a uma ferramenta de apoio à decisão denominada por SuperDecisions ${ }^{5}$. O método AHP tem sido muito usado na tomada de decisões multicritério, fornecendo um quadro abrangente e racional para estruturar um problema de decisão, representar os seus elementos e quantificar os mesmos. Este método permite uma comparação sistemática de alternativas, sendo aplicável a praticamente todas as áreas de tomada de decisão, incluindo a seleção de ferramentas de software (Cruz-Cunha, Silva, Gonçalves, Fernandes, \& Ávila, 2016). Entre as vantagens do método AHP estão (i) a avaliação multicritérios, (ii) a utilização de variáveis linguísticas e (iii) a necessidade de uma análise aprofundada para definir os pesos das várias alternativas de decisão (Vanti, Solana-González, \& Seibert, 2018). Através das classificações finais das ferramentas BPM, o método AHP indicará qual a melhor ferramenta BPM ao longo de todo o ciclo de vida BPM e em cada uma das respetivas fases. O método AHP realizará ciclicamente (duas a duas) a comparação entre as ferramentas BPM e o grau de comparação entre as ferramentas BPM em cada um dos critérios tecnológicos será divido em três níveis: (i) Igual - se uma ferramenta BPM apresenta o mesmo grau de importância (mesmo número de pontos) que a outra ferramenta BPM, nível representado pelo valor "1" na escala do método AHP; (ii) Moderado - se uma ferramenta BPM apresenta um grau de importância moderadamente mais forte (exatamente mais um ponto) que a outra ferramenta BPM, nível representado pelo valor " 3 " na escala do método AHP; e (iii) Forte - se uma ferramenta BPM apresenta um grau de importância mais forte (mais dois pontos) que a outra ferramenta BPM, nível representado pelo valor " 5 " na escala do método AHP.

\footnotetext{
${ }^{5}$ Disponível em: https://www.superdecisions.com/
} 
A partir da abordagem metodológica do método AHP, os resultados da definição final de prioridades são apresentados a seguir para alcançar o objetivo de identificar qual a melhor ferramenta BPM. SuperDecisions fornece diferentes análises priorizadas para os diferentes critérios, bem como o ranking das diferentes alternativas de decisão. As tabelas 13 a 18, produzidas pelo SuperDecisions, incluem as colunas: (i) Graphic (gráfico de barras relativo ao valor na coluna Total), (ii) Alternatives (identificação dos itens em avaliação), (iii) Total (pontuação final de cada item de avaliação), (iv) Normal (valores resultantes da normalização dos valores na coluna Total), (v) Ideal (valores resultantes da divisão dos valores na coluna Total pelo maior valor presente na coluna Total) e (vi) Ranking (identificação da posição dos itens em avaliação) (Creative Decisions Foundation, 2018). Estas tabelas apresentam os resultados obtidos pelas ferramentas BPM e sumarizam o resultado da aplicação do método AHP, no qual é apresentada uma linha por cada item de avaliação (ferramentas BPM). A Tabela 13 apresenta os resultados obtidos pelas ferramentas BPM na fase de Desenho e Análise.

\begin{tabular}{||c||c||c||c||c|c|}
\hline Graphic & Alternatives & Total & Normal & Ideal & Ranking \\
\hline \hline & 1Bonita & 0.2578 & 0.2578 & 0.7357 & 2 \\
\hline \hline & 2ProcessMaker & 0.2179 & 0.2179 & 0.6220 & 3 \\
\hline \hline & 3Bizagi & 0.3504 & 0.3504 & 1.0000 & 1 \\
\hline \hline & 4Alfresco & 0.1739 & 0.1739 & 0.4963 & 4 \\
\hline \hline
\end{tabular}

Tabela 13 - Resultados Obtidos Fase de Desenho e Análise

Na fase de Desenho e Análise, Bizagi obteve a classificação final mais alta e a pontuação máxima nos dez critérios tecnológicos. Alfresco obteve a classificação final mais baixa, embora tenha obtido a pontuação máxima em três dos dez critérios tecnológicos. A Tabela 14 apresenta os resultados obtidos pelas ferramentas BPM na fase de Configuração.

\begin{tabular}{|c||c||c||c||c|c|}
\hline Graphic & Alternatives & Total & Normal & Ideal & Ranking \\
\hline \hline & 1Bonita & 0.2259 & 0.2259 & 0.8264 & 4 \\
\hline \hline & 2ProcessMaker & 0.2502 & 0.2502 & 0.9155 & 3 \\
\hline \hline & 3Bizagi & 0.2506 & 0.2506 & 0.9168 & 2 \\
\hline \hline & 4Alfresco & 0.2733 & 0.2733 & 1.0000 & 1 \\
\hline
\end{tabular}

Tabela 14 - Resultados Obtidos Fase de Configuração

Na fase de Configuração, Alfresco obteve a classificação final mais alta. Bonita obteve a classificação final mais baixa, apesar de ter obtido a pontuação máxima em seis dos onze critérios tecnológicos. ProcessMaker é a única que pontuou nos onze critérios tecnológicos. A Tabela 15 apresenta com os resultados obtidos pelas ferramentas BPM na fase de Execução. 


\begin{tabular}{||c||c|c|c|c|c||}
\hline Graphic & Alternatives & Total & Normal & Ideal & Ranking \\
\hline \hline & 1Bonita & 0.2150 & 0.2150 & 0.6328 & 3 \\
\hline \hline & 2ProcessMaker & 0.2104 & 0.2104 & 0.6193 & 4 \\
\hline \hline & 3Bizagi & 0.3397 & 0.3397 & 1.0000 & 1 \\
\hline \hline & 4Alfresco & 0.2350 & 0.2350 & 0.6917 & 2 \\
\hline
\end{tabular}

Tabela 15 - Resultados Obtidos Fase de Execução

Na fase de Execução, Bizagi obteve a classificação final mais alta e obteve a pontuação máxima nos dez critérios tecnológicos. ProcessMaker obteve a classificação final mais baixa, mesmo tendo a pontuação máxima em cinco dos dez critérios tecnológicos. A Tabela 16 apresenta os resultados obtidos pelas ferramentas BPM na fase de Avaliação.

\begin{tabular}{|c||c||c|c||c|c||}
\hline Graphic & Alternatives & Total & Normal & Ideal & Ranking \\
\hline \hline & 1Bonita & 0.3432 & 0.3432 & 1.0000 & 2 \\
\hline \hline & 2ProcessMaker & 0.1849 & 0.1849 & 0.5387 & 3 \\
\hline \hline & 3Bizagi & 0.3432 & 0.3432 & 1.0000 & 1 \\
\hline \hline & 4Alfresco & 0.1286 & 0.1286 & 0.3747 & 4 \\
\hline \hline
\end{tabular}

Tabela 16 - Resultados Obtidos Fase de Avaliação

Na fase de Avaliação, Bonita e Bizagi obtiveram a classificação final mais alta e a pontuação máxima nos três critérios tecnológicos. Alfresco obteve a classificação final mais baixa, tendo obtido a pontuação máxima em um dos três critérios tecnológicos. A Tabela 17 apresenta os resultados obtidos pelas ferramentas BPM em Administração e Intervenientes.

\begin{tabular}{||c||c||c|c||c|c||}
\hline Graphic & Alternatives & Total & Normal & Ideal & Ranking \\
\hline \hline & 1Bonita & 0.2667 & 0.2667 & 1.0000 & 3 \\
\hline \hline & 2ProcessMaker & 0.2667 & 0.2667 & 1.0000 & 2 \\
\hline \hline & 3Bizagi & 0.2667 & 0.2667 & 1.0000 & 1 \\
\hline \hline & 4Alfresco & 0.2000 & 0.2000 & 0.7500 & 4 \\
\hline \hline
\end{tabular}

Tabela 17 - Resultados Obtidos Administração e Intervenientes

Em Administração e Intervenientes, Bonita, ProcessMaker e Bizagi obtiveram a classificação final mais alta, pois conseguiram a pontuação máxima nos três critérios tecnológicos. Alfresco obteve a classificação final mais baixa, apesar de ter obtido a pontuação máxima em dois dos três critérios tecnológicos. A Tabela 18 apresenta os resultados obtidos pelas ferramentas BPM ao longo do ciclo de vida BPM. 


\begin{tabular}{||c||c||c|c||c|c||}
\hline Graphic & Alternatives & Total & Normal & Ideal & Ranking \\
\hline \hline & 1Bonita & 0.8390 & 0.2602 & 0.8532 & 2 \\
\hline \hline & 2ProcessMaker & 0.7391 & 0.2292 & 0.7516 & 3 \\
\hline \hline & 3Bizagi & 0.9834 & 0.3050 & 1.0000 & 1 \\
\hline \hline & 4Alfresco & 0.6625 & 0.2055 & 0.6737 & 4 \\
\hline \hline
\end{tabular}

Tabela 18 - Resultados Obtidos Ferramentas BPM

Ao longo do ciclo de vida BPM, Bizagi obteve a classificação final mais alta e com a pontuação máxima em trinta e três dos trinta e sete critérios tecnológicos. Alfresco obteve a classificação final mais baixa, embora tenha obtido a pontuação máxima em vinte e um dos trinta e sete critérios tecnológicos.

\section{Conclusões}

O trabalho efetuado teve uma componente inicial muito importante, relacionada com a escolha de qual o ciclo de vida BPM, que permitiu definir a base da estrutura de avaliação. Os resultados obtidos através da classificação das ferramentas BPM demonstram que o método de avaliação permite distinguir entre as diferentes ferramentas BPM e apresenta valores díspares em cada uma das ferramentas, permitindo realçar os seus aspetos mais relevantes. Ao longo da investigação da documentação técnica das ferramentas BPM identificaram-se os seus principais conceitos e critérios tecnológicos, uma vez que a realização da classificação das ferramentas BPM segundo a documentação técnica requer algum conhecimento prévio dos conceitos e critérios tecnológicos utilizados em cada ferramenta BPM. De acordo com a ferramenta BPM o mesmo conceito pode ter designações diferentes, por exemplo, os intervenientes do processo de negócio na ferramenta BPM Bonita são designados por Atores (Actors) enquanto na ferramenta BPM Bizagi são designados por Executantes (Performers).

A análise de resultados discutida neste artigo poderá ser útil para a tomada de decisão entre uma destas ferramentas BPM: Bonita, ProcessMaker, Bizagi e Alfresco Process Services. De acordo com resultados obtidos, a ferramenta BPM Bizagi posicionase como a ferramenta BPM que reúne um maior número de aspetos positivos, no entanto as organizações têm sempre de verificar se esta será a ferramenta BPM que melhor se adapta às suas necessidades organizacionais. Conclui-se convictamente que, à semelhança destas quatro ferramentas BPM, uma ferramenta BPM que implementa os conceitos abordados ao longo deste artigo é sem dúvida uma maisvalia para as organizações. A utilização de ferramentas BPM permite gerir, otimizar e automatizar os processos de negócio ao longo de todo o processo, possibilitando interações com diferentes intervenientes, assim como reagir rapidamente as solicitações, do mercado tornando as organizações mais competitivas que os seus concorrentes. A aplicação da estrutura de avaliação definida neste artigo em diferentes ferramentas BPM irá contribuir com mais informação tecnológica para esta área do conhecimento. 


\section{Referências}

Bischoff, B., \& van Dinther, C. (2016). Workflow Management Systems an analyis of current open source products. Digital Enterprise Computing 2016. Lecture Notes in Informatics, 2016, 147-160. Obtido de http://subs.emis.de/LNI/ Proceedings/ Proceedings258/article19.html

Craggs, S. (2011). Comparing BPM from IBM, Software AG and Pegasystems. Obtido de http://lustratus.com/product/comparing-bpm-from-pegasystems-ibm-and-tibco/

Creative Decisions Foundation (2018). Super Decisions: AHP and ANP. Obtido de http://www.superdecisions.com/method/

Cruz-Cunha, M. M., Silva, J. P., Gonçalves, J. J., Fernandes, J. A., \& Ávila, P. S. (2016). ERP Selection using an AHP-based Decision Support System. Information Resources Management Journal, 29(4), 65-81. https://doi.org/10.4018/IRMJ.2016100105

Dunie, R., Kerremans, M., Baker, V. L., \& Wong, J. (2017). Magic Quadrant for Intelligent Business Process Management Suites. Obtido de https://www.gartner.com/en/ documents $/ 3818763$

Hill, J. B., \& Sinur, J. (2010). Magic Quadrant for Business Process Management Suites. Obtido de https://www.gartner.com/en/documents/1453527

Hill, J. B., Sinur, J., Flint, D., \& Melenovsky, M. J. (2006). Gartner's Position on Business Process Management. Obtido de https://www.gartner.com/en/ documents/489533

Hook, G. (2011). Business Process Modeling and Simulation. Proceedings of the 2011 Winter Simulation Conference (WSC), 773-778. https://doi.org/10.1109/ WSC.2011.6147804

Ko, R. K. L. (2009). A Computer Scientist's Introductory Guide to Business Process Management (BPM). Crossroads, 15(4), 11-18. https://doi. org/10.1145/1558897.1558901

Marques, R. P., \& Guerreiro, S. (2016). Mecanismo de controlo para a frente orientado ao risco como garantia da conformidade da execução de processos de negócio. RISTI - Revista Iberica de Sistemas e Tecnologias de Informacao, 2016(20), 34-47. https://doi.org/10.17013/risti.20.34-47

Meidan, A., García-García, J. A., Escalona, M. J., \& Ramos, I. (2016). A Survey on Business Processes Management Suites. Computer Standards and Interfaces, 51, 71-86. https://doi.org/10.1016/j.csi.2016.06.003

OMG (2011). Business Process Model and Notation (BPMN) Version 2.o. Business. Obtido de https://www.omg.org/spec/BPMN/2.0/About-BPMN/

Peralta, M., Salgado, C., Montejano, G., \& Riesco, D. (2016). Las Redes Sociales y la Nube: Un nuevo Paradigma para los Procesos de Negocio. RISTI - Revista Iberica de Sistemas e Tecnologias de Informacao, 2016(18), 66-82. https://doi. org/10.17013/risti.18.66-82 
Rademakers, T. (2012). Activiti in Action: Activiti in Action: Executable business processes in BPMN 2.O. Shelter Island: Manning Publications.

Richardson, C., \& Miers, D. (2013). The Forrester Wave: BPM Suites, Q1 2013. Obtido de https://www.forrester.com/report/The+Forrester+Wave+BPM+ Suites+Q1+2013/-/E-RES88581

Sousa, M., Lopes, N., Ribeiro, O., \& Silva, J. (2018). Evaluation of BPM tools open source/freeware. In 2018 13th Iberian Conference on Information Systems and Technologies (CISTI). IEEE. https://doi.org/10.23919/CISTI.2018.8398642

Van der Aalst, W. M. P. (2013). Business Process Management: A Comprehensive Survey. ISRN Software Engineering, 2013, 1-37. https://doi.org/10.1155/2013/507984

Van der Aalst, W. M. P., ter Hofstede, A. H. M., \& Weske, M. (2003). Business Process Management: A Survey. In Business Process Management. BPM 2003. Lecture Notes in Computer Science (Vol. 2678, pp. 1-12). Berlin: Springer.

Van der Aalst, W. M. P., \& van Hee, K. (2004). Workflow Management: Models, Methods and Systems. US: The MIT Press.

Vanti, A. A., Solana-González, P., \& Seibert, R. (2018). Corporate governance and IT corporate governance using the analytic hierarchy process in creating value. RISTI - Revista Iberica de Sistemas e Tecnologias de Informacao, 2018(27), 86-108. https://doi.org/10.17013/risti.27.86-108

Weske, M. (2012). Business Process Management: Concepts, Languages, Architectures (2. ${ }^{\mathrm{a}}$ ed.). Berlin: Springer. 